\title{
Transient effects in the backscattered current of a Luttinger liquid
}

\author{
Mariano J. Salvay, Hugo A. Aita, and Carlos M. Naón \\ Departamento de Física, Facultad de Ciencias Exactas, Universidad Nacional de La Plata and IFLP-CONICET, \\ CC 67, 1900 La Plata, Argentina
}

(Received 14 December 2009; published 5 March 2010)

\begin{abstract}
We study the backscattered current in a Luttinger liquid in the presence of a pointlike weak impurity switched on at finite time, taking into account finite-temperature effects. We show how the well-known results for a static impurity are distorted. We derive a dimensionless parameter $\tau_{R}$ as a function of the electron-electron interaction and the temperature, such that for $\tau_{R}<1(>1)$ the switching process is relevant (irrelevant). Our results suggest the possibility of determining the value of the Luttinger parameter $K$ through time measurements in transport experiments at fixed voltage.
\end{abstract}

DOI: 10.1103/PhysRevB.81.125406 PACS number(s): 71.10.Pm, 73.63.Nm, 05.30.Fk, 72.10.Bg

\section{INTRODUCTION}

Quantum transport in novel one-dimensional (1D) materials, such as quantum wires and carbon nanotubes, ${ }^{1}$ is one of the most active areas of present research in condensed-matter physics. Recent experiments have confirmed some of the striking effects that characterize the Luttinger liquid (LL) picture of 1D nanostructures, ${ }^{2}$ such as spin-charge separation $^{3}$ and charge fractionalization. ${ }^{4}$ One central issue in all experiments is the determination of Luttinger exponents that depend on the rigidity constant $K$, which parametrizes electron-electron (e-e) interactions. These exponents have been measured in pioneering tunneling experiments. ${ }^{5}$ However, some doubts still remain in the determination of $K$ due to the fact that dynamical Coulomb blockade also leads to power laws in systems surrounded by Ohmic devices. ${ }^{6}$ Moreover, it has been shown that environmental resistance can also contribute to the measured value of $K{ }^{7}$ It is then very important to conceive alternative ways to measure $K$. To a large extent, quantum transport is highly nontrivial due to the influence of a variety of combined effects produced by impurities, junctions, contacts, etc. In particular, in the study of impurities in Luttinger liquids, an observable of special interest is the backscattered current $I_{b s}$. For a pointlike static impurity, $I_{b s}$ opposes the background current and goes as $V^{2 K-1}$, where $V$ is the bias voltage. ${ }^{8}$ The effects of finite temperature and finite length of the quantum wire lead to characteristic nonmonotonic parameter dependencies of the current and yield a rich structure in the noise spectrum which depends on $K .{ }^{9}$ For a time-dependent oscillatory impurity, the current grows and the conductance of a one-channel quantum wire is greater than its background value $e^{2} / h$ for strong repulsive interaction $(K<1 / 2) .{ }^{10,11}$ More recently, the effect of several time-dependent impurities was considered at zero temperature and infinite length. ${ }^{12,13}$ When temperature is taken into account, for two oscillating barriers, the previous zero-temperature results are distorted; the behavior of the backscattered current and the change in the differential conductance depend on different regimes which can be established as functions of the frequency, the temperature, and the separation between the impurity potentials. ${ }^{14}$

One aspect that has been seldom explored in the context of the Luttinger liquid is its response to a sudden switch of an interaction of the system with an external field. As an example, the total-energy density of a Tomonaga-Luttinger liquid in the presence of a forward-scattering time-dependent impurity switched on at finite time has been computed exactly, distinguishing two well-defined regimes in terms of the relationship between the frequency of the perturbation and the electron energy. This study allowed us to determine a time interval in which the finite-time switching process is relevant. $^{15}$

In this paper we examine the effect of a transient process on transport properties of a Luttinger liquid. When a local barrier, which can be considered as a backscattering impurity, is turned on at a finite time $t_{0}$, a backscattered current is produced. Let us stress that such a barrier can be created, for instance, in a single-walled carbon nanotube by applying a voltage to a narrow metal gate electrode ${ }^{16}$ (see Fig. 1). We obtain an analytical expression for $I_{b s}$ as a function of time. The envelope of this function decays in a way that crucially depends on e-e interactions, i.e., on the $K$ parameter. This allows us to find a simple relation between the decay process and the value of $K$. Thus, we show that $K$ could be determined by measuring time intervals within the reach of recently developed pump-probe techniques with femtosecondattosecond time resolutions. ${ }^{17}$ Then, in contrast to the conventional techniques used up to now to measure $K$, based on the study of stationary transport properties for varying voltages, we indicate an alternative way based on the analysis of the transient current for fixed voltage. Apart from its interest in the context of strongly correlated electronic systems, our studies could be relevant in the area of cold atomic

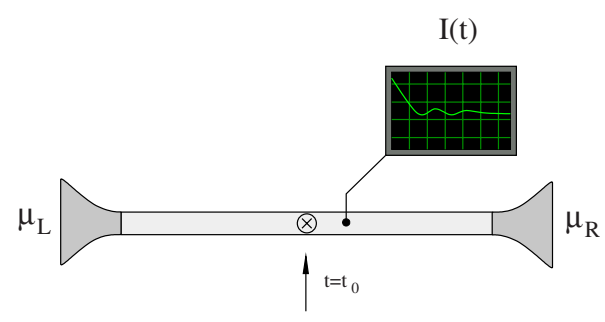

FIG. 1. (Color online) The figure shows a quantum wire coupled adiabatically to two reservoirs with different chemical potentials, with a backscattering impurity switched on at time $t=t_{0}$. The current $I(t)$ is measured as a function of time. 
gases, where quantum quenches are being intensively investigated. $^{18}$

The work is organized as follows. In Sec. II we present the model and review the calculation of the backscattered current in terms of a vacuum expectation value (v.e.v.) of exponentials of bosonic fields. In Secs. III and IV we present the results obtained at zero and finite temperatures, respectively. We define a dimensionless relaxation parameter $\tau_{R}$ as a function of the temperature and of the electron-electron interaction. Finally, we analyzed and summarize our results in Sec V.

\section{MODEL}

We consider a long and clean LL adiabatically coupled to two electrodes with different chemical potentials, such that $\mu_{L}-\mu_{R}=e V$. We will restrict our study to the case in which the electrodes are held at the same temperature. This condition is very important in order to apply standard bosonization techniques. ${ }^{19}$ Indeed, as was very recently explained, ${ }^{20}$ standard bosonization is expected to work well only in special situations corresponding to small deviations from equilibrium. The nontrivial ingredient of our model is a barrier of constant height (an externally controlled impurity) that is turned on at a finite time $t_{0}$, producing backscattering of incident waves. Under these conditions we model the LL in terms of the following Lagrangian density: ${ }^{13}$

$$
L=L_{0}+L_{i m p}
$$

where

$$
L_{0}=\frac{1}{2} \Phi(x, t)\left(v^{2} \frac{\partial^{2}}{\partial_{x}^{2}}-\frac{\partial^{2}}{\partial_{t}^{2}}\right) \Phi(x, t)
$$

describes a spinless Tomonaga-Luttinger liquid with renormalized velocity $v$ and

$$
\begin{aligned}
L_{i m p}= & -\frac{g_{B}}{\pi \hbar \Lambda} \delta\left(x-x_{0}\right) \Theta\left(t-t_{0}\right) \\
& \times \cos \left[2 k_{F} x / \hbar+2 \sqrt{\pi K v} \Phi(x, t)+e V t / \hbar\right]
\end{aligned}
$$

represents the scattering of spinless electrons with the external barrier at the point $x_{0}$ and switched on at the time $t_{0}$, with a coupling amplitude $g_{b}$. $V$ is the external voltage applied to the quantum wire and $K$ measures the strength of the electron-electron interactions. For repulsive interactions $K$ $<1$ and for noninteracting electrons $K=1 . \Lambda$ is a shortdistance cutoff. In the above expression we only take into account backscattering between electrons and impurities, because the forward scattering does not change the transport properties studied here, at least, in the lowest order of the perturbative expansion in the couplings.

In the absence of the impurity, the background current is $I_{0}=e^{2} V / h$. When the impurity is acting the total current is $I=I_{0}-I_{b s}$. The operator associated with the backscattered current is defined as ${ }^{13}$
$\hat{I}_{b s}(t)=\frac{g_{B} e}{\pi \hbar \Lambda} \Theta\left(t-t_{0}\right) \sin \left[2 k_{F} x_{0} / \hbar+2 \sqrt{\pi K v} \hat{\Phi}\left(x_{0}, t\right)+e V t / \hbar\right]$.

The backscattered current at any time $t$ is given by

$$
I_{b s}(t)=\left\langle 0\left|S(-\infty ; t) \hat{I}_{b s}(t) S(t ;-\infty)\right| 0\right\rangle,
$$

where $\langle 0|$ denotes the initial state and $S$ is the scattering matrix, which to the lowest order in the coupling $g_{B}$ is given by

$$
S(t ;-\infty)=1-i \int_{-\infty}^{\infty} d x \int_{-\infty}^{t} L_{i m p}\left(t^{\prime}\right) d t^{\prime}
$$

When one inserts Eq. (6) in Eq. (5) one finds several terms of the form

$$
A_{\alpha, \beta}=\left\langle 0\left|\exp \left[2 i \alpha \sqrt{\pi K v} \hat{\Phi}\left(x, t^{\prime}\right)\right] \exp [-2 i \beta \sqrt{\pi K v} \hat{\Phi}(x, t)]\right| 0\right\rangle,
$$

with $\alpha, \beta= \pm 1$. This kind of v.e.v. of vertex operators has been computed many times in the literature. It is well known that $A_{\alpha,-\alpha}=0$. Thus, the building block of our computation is $A_{\alpha, \alpha}$. Let us sketch the calculational procedure for $\alpha=1$. Using the Baker-Haussdorff formula and taking into account that the commutator of the fields is a $c$ number, we can write

$$
\begin{aligned}
A_{1,1}= & \left\langle 0\left|\exp \left\{2 i \sqrt{\pi K v}\left[\hat{\Phi}\left(x, t^{\prime}\right)-\hat{\Phi}(x, t)\right]\right\}\right| 0\right\rangle \\
& \times \exp \left\{2 \pi K v\left[\hat{\Phi}\left(x, t^{\prime}\right), \hat{\Phi}(x, t)\right]\right\} .
\end{aligned}
$$

At this point we observe that the v.e.v. of the exponential in the first factor above can be written as the exponential of a v.e.v.,

$$
\begin{aligned}
& \left\langle 0\left|\exp \left\{2 i \sqrt{\pi K v}\left[\hat{\Phi}\left(x, t^{\prime}\right)-\hat{\Phi}(x, t)\right]\right\}\right| 0\right\rangle \\
& \quad=\exp \left\{-2 \pi K v\left\langle 0\left|\left[\hat{\Phi}\left(x, t^{\prime}\right)-\hat{\Phi}(x, t)\right]^{2}\right| 0\right\rangle\right\} .
\end{aligned}
$$

Now, in order to explicitly evaluate the previous expressions we need the Keldysh ${ }^{21}$ lesser function $G^{<}$given by

$$
\begin{aligned}
\left\langle 0\left|\hat{\Phi}\left(x, t^{\prime}\right) \hat{\Phi}(x, t)\right| 0\right\rangle= & i G^{<}\left(x, t ; x, t^{\prime}\right) \\
= & \frac{1}{2 \pi} \int d p d \omega e^{-i \omega\left(t-t^{\prime}\right)}[\theta(-\omega) \\
& \left.+n_{B}(|\omega|)\right] \delta\left(\omega^{2}-v^{2} p^{2}\right),
\end{aligned}
$$

where $n_{B}(|\omega|)=\left(e^{\hbar|\omega| / k_{B} T}-1\right)^{-1}$. Putting all this together we finally obtain

$$
\begin{gathered}
\left\langle 0\left|\exp \left[i 2 \sqrt{\pi K v} \hat{\Phi}\left(x, t^{\prime}\right)\right] \exp [-i 2 \sqrt{\pi K v} \hat{\Phi}(x, t)]\right| 0\right\rangle \\
=\frac{\left(\Lambda \pi k_{b} T / \hbar v\right)^{2 K} \exp \left\{i \pi K \operatorname{sgn}\left[t-t^{\prime}\right]\right\}}{\left|\sinh \left[\pi k_{b} T\left(t-t^{\prime}\right) / \hbar\right]\right|^{2 K}},
\end{gathered}
$$

where sgn is the sign function.

\section{RESULTS AT ZERO TEMPERATURE}

First of all, we compute Eq. (5) at zero temperature and with the impurity switched on at time $-\infty$, 


$$
I_{b s}(t,-\infty)=\frac{g_{B}^{2} e \Lambda^{2 K-2}}{2 \pi \hbar^{2} v^{2 K} \Gamma[2 K]}\left|\frac{e V}{\hbar}\right|^{2 K-1} \operatorname{sgn}[V] .
$$

This corresponds to the well-known case of a static impurity, ${ }^{8}$ where the backscattered current goes as $V^{2 K-1}$. We note that, for $K<1 / 2$, the backscattered current becomes large when $V$ decreases. Hence, the perturbative expansion in powers of $g_{B}$ breaks down when $V \rightarrow 0$. Using a scaling analysis we can estimate that this expansion is valid when $\left(g_{B} / \hbar v\right)(\Lambda e V / \hbar v)^{K-1} \ll 1$. We emphasize that expression (12) does not include the case $V=0$, where the current is zero, too. All these statements imply that the current must be a nonmonotonic function of $V$. In order to determine this function one has to go beyond the lowest-order perturbative results of this work.

Now, we compute the backscattered current when the impurity is switched on at a finite time $t_{0}$,

$$
\begin{aligned}
I_{b s}\left(t, t_{0}\right)= & \frac{\Theta(\tau) \Gamma[2 K] \tau^{2-2 K}}{\Gamma[K] \Gamma[2-K]} \\
& \times_{1} F_{2}\left[1-K ; 3 / 2,2-K ;-(\tau / 2)^{2}\right] I_{b s}(t,-\infty),
\end{aligned}
$$

where ${ }_{1} F_{2}$ is the generalized hypergeometric function and $\tau$ $=e V\left(t-t_{0}\right) / \hbar$ is a dimensionless scaling parameter, so that $\tau \gg 1(\ll 1)$ represents a large (short) time elapsed since the time $t_{0}$. This expression is the first nontrivial result of this work. We have obtained an analytical expression for the backscattered current taking into account the effect of the nonadiabatic switching of the barrier. In the long-time regime this current approaches the stationary value $I_{b s}(t,-\infty)$.

In order to perform a quantitative analysis of the transient process, which is now accessible due to the introduction of an abrupt triggering mechanism, we found useful to evaluate the relative change between currents turned on at times $t_{0}$ and $-\infty, f(\tau)=\left[I_{b s}\left(t, t_{0}\right) / I_{b s}(t,-\infty)\right]-1$. This is a damped oscillatory function of $\tau$ with period $2 \pi$ and relative maxima in $\tau=(2 n+1) \pi$ with $n$ natural. As expected, $f(\tau)$ goes to zero when $\tau \rightarrow \infty$, i.e., when the current is the one corresponding to a simple static impurity acting at all times. At this point, in order to have a more intuitive picture of the transient process, we define a reference value of $\tau, \tau_{R}$, that enables us to identify time scales for which the transitory stage is relevant or not. To this end it seems natural to examine the way in which the relative maxima of $f(\tau)$ decrease as $\tau$ increases. Since the first maximum is located at $\tau=\pi$, it is a very good approximation to use the following asymptotic $(\tau \gg 1)$ expression for $f(\tau)$ :

$$
f(\tau) \approx A(\tau, K) \cos [\tau+\pi],
$$

where $A(\tau, K)=\tau^{-2 K} / \cos [\pi K] \Gamma[1-2 K]$ is the envelope of a damped harmonic oscillation. Since the change in the backscattered current due to the sudden switching behaves as $\tau^{-2 K}$, we conclude that the relaxation of the system is faster for small electron-electron interaction (for the sake of clarity, let us stress that this relaxation characterizes the transition of the backscattering current between two off-equilibrium regimes). We thus find an explicit connection between electron interactions and the switching time of the externally con- trolled barrier: the stronger the correlations, the longer the persistence of the nonadiabatic effect. Formula (14) provides a direct way of defining a dimensionless relaxation parameter $\tau_{R}$, such that for $\tau_{R}<1(>1)$ the switching process is relevant (irrelevant). We define $\tau_{R}$ as the value of $\tau$ such that $A(\tau, K)=1 / r(r>1)$, which means that for $\tau>\tau_{R}$ the value of $I_{b s}\left(t, t_{0}\right)$ differs by less than $(100 / r) \%$ from the current obtained when the switching process is not taken into account. To be definite, in the following we set $r=10$. We then get

$$
\tau_{R}=\left(\frac{10}{\cos [\pi K] \Gamma[1-2 K]}\right)^{1 / 2 K} .
$$

The solid line in Fig. 3 shows the behavior of $\tau_{R}$ as a function of $K$ at zero temperature. We observe that $\tau_{R}$ grows as $10^{1 /(2 K)}$ for strong electron-electron interactions $(K \rightarrow 0)$. For weak interactions $(K \rightarrow 1)$ the effect of the sudden switching is negligible; $\tau_{R}$ goes to zero as $(1-K)^{1 / 2}$.

At this point it is useful to recall that the measurable time in this process is the difference $t-t_{0}$. The time interval that it takes the backscattered current to reach a value that differs in exactly $10 \%$ from the steady current is $t_{R}-t_{0}=\hbar \tau_{R} / \mathrm{eV}$. Using the results shown in Fig. 1 for the zero-temperature case, which relate values of $K$ with values of $\tau_{R}$, we can estimate $t_{R}-t_{0}$. Bias voltages usually applied in experiments with nanodevices range from $\mu \mathrm{V}$ 's to $\mathrm{mV}$ 's. On the other hand, typical interactions take values in the range $0.25<K<0.75$. $^{3}$ With these data we obtain $10^{-11}<t_{R}-t_{0}<10^{-7} \mathrm{~s}$, which are time intervals that could be measured with recently developed pump-probe techniques which reach femtosecondattosecond time resolutions. ${ }^{17}$

The relationship between the relaxation process and the strength of Coulombic electron correlations revealed in our analysis might provide an alternative way to determine the Luttinger parameter $K$ through time measurements. The total current after the switching as a function of time is $I(t)$ $=I(\infty)+C t^{-2 K} \cos [\Omega t]$, where $\Omega=e V / \hbar, C$ is a constant, and $I(\infty)$ is the stationary current for a static barrier. A determination of the current as a function of time with a temporal resolution smaller than $2 \pi \hbar / \mathrm{eV}$ is a direct method to obtain the exponent of the temporal decay, and then the $K$ value of the quantum wire. We emphasize that this proposed method is performed at constant source-drain voltage.

In Fig. 2 we illustrate the experimental determination of $K$ according to the ideas mentioned above. The solid line represents the measurement of the total current $I(t)$ after the switching of the impurity. All parameters involved in this plot (the voltage and the strength of the barrier) are within the range of experimental reach. The temporal resolution corresponding to this hypothetical measurement is on the order of the picosecond, which is accessible with the already mentioned state of the art pump-probe techniques. The next step is to determine the envelope of the curve, given by the dashed line in Fig. 2. This envelope can be fitted with the $t^{-2 K}$ law in order to find $K$. This procedure, which we depict here for $T=0$, can be extended for finite temperatures, as we will show in the next section. The power law $t^{-2 K}$ is a very good approximation for $T \ll e V / \pi k_{B}$. For source-drain voltages on the order of mV's, which we used in the case of Fig. 2, this corresponds to $T \ll 10 \mathrm{~K}$. 


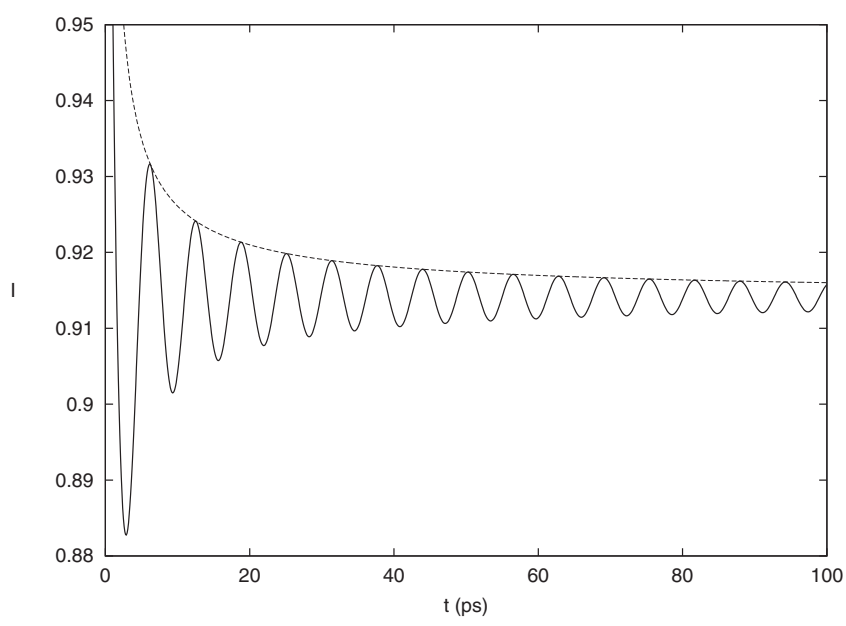

FIG. 2. Example of an experimental plot of $I$ as a function of $t$, leading to the determination of $K$. We have set $\frac{e V}{t}=10^{12}(1 / \mathrm{s})$ (corresponding to $V \simeq \mathrm{mV}), g_{B} / \hbar v=(0.1)^{1 / 2}$, and $\frac{\Lambda e V}{\hbar v}=1 . I$ is measured in units of $e^{2} V / h$. For this particular case, using the envelope (dashed line) one obtains $K=0.4$.

\section{RESULTS AT FINITE TEMPERATURE}

Taking into account that experiments are performed at very low but finite temperatures, in this section we show how our results are affected by thermal effects. Using expression (11) for vacuum expectation values of exponentials of bosonic fields, we can extend the results of Sec. III in a straightforward way. The backscattered current with the impurity switched on at time $-\infty$ (static impurity) becomes

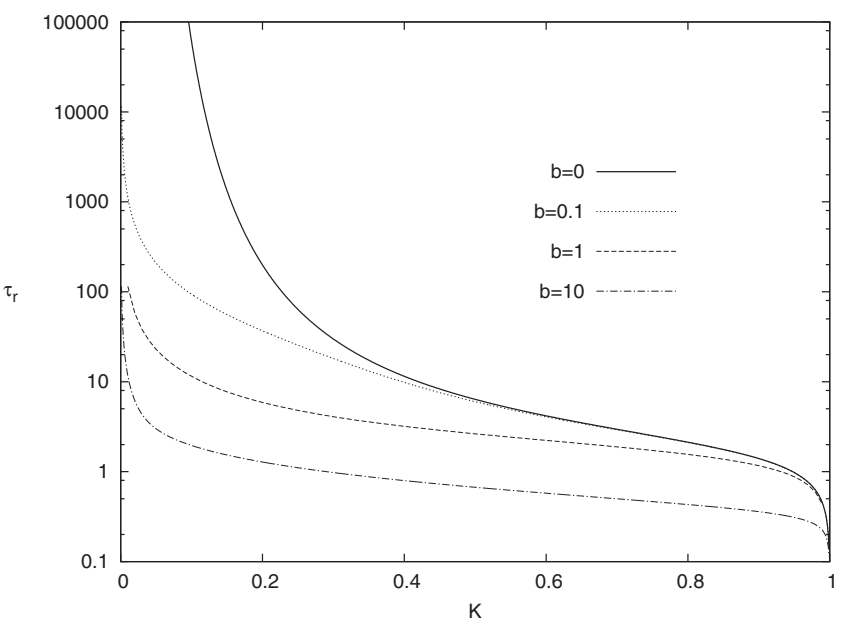

FIG. 3. Dimensionless relaxation parameter $\tau_{R}$ as a function of $K$ and for different values of $b$.

$$
\begin{aligned}
I_{b s}(t,-\infty)= & \frac{g_{B}^{2} e \Lambda^{2 K-2}}{2 \pi^{2} \hbar^{2} v^{2 K} \Gamma[2 K]}\left(2 \pi k_{b} T / \hbar\right)^{2 K-1} \\
& \times \Gamma\left[K-\frac{i}{2 b}\right] \Gamma\left[K+\frac{i}{2 b}\right] \sinh \left[\frac{\pi}{2 b}\right],
\end{aligned}
$$

where $b=\pi k_{b} T / e V$ is a dimensionless parameter that characterizes the scale regime in temperature of the system: $b \gg 1$ $(\ll 1)$ is the high- (low-) temperature regime, for fixed voltage. The perturbative expansion is valid for low temperature when $\left(g_{B} / \hbar v\right)(\Lambda e V / \hbar v)^{K-1} \ll 1$ and for high temperature when $\left(g_{B} / \hbar v\right)\left(\Lambda k_{b} T / \hbar v\right)^{K-1} \ll 1$.

The backscattered current when the impurity is switched on at a finite time $t_{0}$ is

$$
I_{b s}\left(t, t_{0}\right)=\left\{1+\frac{i \Theta(\tau) \Gamma[2 K] \sin [\pi K]\left(B\left[\exp [2 b \tau], \frac{-i}{2 b}, 1-2 K\right]-*\right)}{\Gamma\left[\frac{-i}{2 b}-K\right] \Gamma\left[\frac{i}{2 b}-K\right] \sinh \left[\frac{\pi}{2 b}\right]}\right\} I_{b s}(t,-\infty),
$$

where $B$ is the incomplete beta function and * indicates the conjugate of the precedent term. This is the analytical generalization of Eq. (13) at finite temperature. As in the former case, the relative change between currents turned on at times $t_{0}$ and $-\infty$ is a damped oscillatory function of $\tau$ with the same period and position of relative maxima as before. Using the asymptotic approximation, we obtain the following expression for the envelope of the damped oscillation:

$A(\tau, K, b)$

$$
\approx \frac{4 b \Gamma[2 K] \sin [\pi K]}{(2 \sinh [b \tau])^{2 K} \Gamma\left[K-\frac{i}{2 b}\right] \Gamma\left[K+\frac{i}{2 b}\right] \sinh \left[\frac{\pi}{2 b}\right]} .
$$

Thus, the change in the backscattered current due to the sudden switching at finite temperature behaves as $\sinh [b \tau]^{-2 K}$ $=\sinh \left[\pi k_{b} T\left(t-t_{0}\right) / \hbar\right]^{-2 K}$. For high temperatures $(b \gg 1)$ the decay is faster and becomes exponential: $A(\tau, K, b)$ $\approx 8 b^{2} \Gamma[2 K] \sin [\pi K] \exp [-2 K b \tau] / \Gamma[K]^{2}$.

Experimentally, the high-temperature regime can be accessed for low voltages. Temperatures involved in the crossover regime $(b \simeq 1)$ are $T \simeq 10 \mathrm{~K}$ and $T \simeq 0.01 \mathrm{~K}$, for applied voltages of order $1 \mathrm{mV}$ and $1 \mu \mathrm{V}$, respectively. In general, if we know the temperature of the system, a method completely analogous to the one described at the end of the previous section can be established to obtain the parameter $K$. The total current after the switching is $I(t)=I(\infty)$ $+C \sinh \left[\pi k_{b} T\left(t-t_{0}\right) / \hbar\right]^{-2 K} \cos [e V t / \hbar]$. Thus, the value of $K$ can be obtained by measuring the current as a function of time at fixed voltage. 
As in the case of zero temperature, we can define a dimensionless relaxation parameter $\tau_{R}$ in the same fashion, i.e., by determining the value of $\tau$ such that $A(\tau, K, b)=0.1$. We obtain for $\tau_{R}$ the following general expression as a function of temperature and electron-electron interaction strength:

$\tau_{R}=\frac{1}{2 b} \operatorname{arcsinh}\left[\left(\frac{40 b \Gamma[2 K] \sin [\pi K]}{\Gamma\left[K-\frac{i}{2 b}\right] \Gamma\left[K+\frac{i}{2 b}\right] \sinh \left[\frac{\pi}{2 b}\right]}\right)^{1 / 2 K}\right]$.

Figure 3 shows the dimensionless relaxation parameter $\tau_{R}$ of the system as a function of $K$, for different values of temperature. We observe that the distortion in the backscattered current caused by the transient process is more important for high electron-electron interactions $(K \ll 1)$ and in the regime of zero or low temperature $(b \ll 1)$, where $\tau_{R} \gg 1$. In this case the relaxation is very slow and the sudden switching changes significantly the value of the backscattered current for a long time. If $b$ and $K$ grow, this effect tends to disappear. For high temperatures, the switching effect becomes irrelevant.

\section{CONCLUSIONS}

We have theoretically analyzed the consequences of a sudden switch of a tunnel barrier on transport properties, in a long and clean LL. In particular we studied the behavior of the backscattered current $I_{b s}$ in a system subjected to a bias voltage $V$. The backscattering time-dependent impurity is assumed to be pointlike and weak. Under these conditions, using bosonization and our result (11) for the expectation value of exponentials of bosonic operators, we obtained an analytical expression for $I_{b s}$ as a function of $\tau=e V\left(t-t_{0}\right) / \hbar$
( $t_{0}$ is the instant at which the barrier is switched on), $b$ $=\pi k_{b} T / \mathrm{eV}$, and the Luttinger parameter $K$ [see formula (17)]. At long times $t \gg t_{0}, I_{b s}\left(t, t_{0}\right)$ reaches a steady-state value that coincides with the value of the current corresponding to a static impurity (a barrier switched on at an infinitely remote instant in the past) $I_{b s}(t,-\infty)$. By carefully examining the way $I_{b s}\left(t, t_{0}\right)$ approaches $I_{b s}(t,-\infty)$ as time goes by, we intuitively characterized the transient process defining a dimensionless relaxation parameter $\tau_{R}$. Employing an asymptotic expression for the exact solution (17), we obtained a simple expression for $\tau_{R}$ as a function of $b$ and $K$ [see formula (19) and Fig. 2]. From this result one concludes that, for fixed bias, $\tau_{R}$ grows with decreasing $K$, meaning that transient effects on the current are expected to last longer when the electron correlation is higher. In fact, $\tau_{R} \rightarrow 0$ for a free system $(K \rightarrow 1)$. One can also see that the transitory effect is more pronounced for low temperatures $(b \ll 1)$.

Finally, we would like to stress that our study could have some interesting experimental applications. Indeed, from our main result (17), and taking into account that the total current after the switching is $I(t)=I_{0}-I_{b s}\left(t, t_{0}\right)$, we obtained $I(t)$ $=I(\infty)+C \sinh \left[\pi k_{b} T\left(t-t_{0}\right) / \hbar\right]^{-2 K} \cos [e V t / \hbar]$. This means that, at fixed temperature and bias voltage, the value of $K$ of a $1 \mathrm{D}$ structure could be determined by performing measurements of the total current as a function of time, after turning on a tunnel barrier through a localized gate voltage, following, for instance, the techniques of Ref. 16.

\section{ACKNOWLEDGMENTS}

This work was partially supported by Universidad Nacional de La Plata (Argentina) and Consejo Nacional de Investigaciones Científicas y Técnicas, CONICET (Argentina). The authors are grateful to M. Di Ventra for a helpful e-mail exchange and to A. Iucci for useful comments.
${ }^{1}$ T. Giamarchi, Quantum Physics in One Dimension (Clarendon Press, Oxford, 2004); M. Di Ventra, Electrical Transport in Nanoscale Systems (Cambridge University Press, Cambridge, England, 2008); Y. V. Nazarov and Y. M. Blanter, Quantum Transport (Cambridge University Press, Cambridge, England, 2009).

${ }^{2}$ J. Voit, Rep. Prog. Phys. 58, 977 (1995).

${ }^{3}$ O. M. Auslaender, H. Steinberg, A. Yacoby, Y. Tserkovnyak, B. I. Halperin, K. W. Baldwin, L. N. Pfeiffer, and K. W. West, Science 308, 88 (2005); Y. Jompol, C. J. B. Ford, J. P. Griffiths, I. Farrer, G. A. C. Jones, D. Anderson, D. A. Ritchie, T. W. Silk, and A. J. Schofield, ibid. 325, 597 (2009).

${ }^{4}$ H. Steinberg, G. Barak, A. Yacoby, L. N. Pfeiffer, K. W. West, B. I. Halperin, and K. Le Hur, Nat. Phys. 4, 116 (2008).

${ }^{5}$ M. Bockrath, D. H. Cobden, J. Lu, A. G. Rinzler, R. E. Smalley, L. Balents, and P. L. McEuen, Nature (London) 397, 598 (1999); Zh. Yao, H. W. Ch. Postma, L. Balents, and C. Dekker, ibid. 402, 273 (1999).

${ }^{6}$ M. H. Devoret, D. Esteve, H. Grabert, G.-L. Ingold, H. Pothier, and C. Urbina, Phys. Rev. Lett. 64, 1824 (1990).
${ }^{7}$ I. Safi and H. Saleur, Phys. Rev. Lett. 93, 126602 (2004).

${ }^{8}$ C. L. Kane and M. P. A. Fisher, Phys. Rev. B 46, 15233 (1992).

${ }^{9}$ F. Dolcini, B Trauzettel, I. Safi, and Hermann Grabert, Phys. Rev. B 71, 165309 (2005).

${ }^{10}$ D. E. Feldman and Y. Gefen, Phys. Rev. B 67, 115337 (2003).

${ }^{11}$ F. Cheng and G. Zhou, Phys. Rev. B 73, 125335 (2006).

${ }^{12}$ A. Agarwal and D. Sen, Phys. Rev. B 76, 035308 (2007).

${ }^{13}$ D. Makogon, V. Juricic, and C. Morais Smith, Phys. Rev. B 74, 165334 (2006).

${ }^{14}$ M. J. Salvay, Phys. Rev. B 79, 235405 (2009).

${ }^{15}$ C. M. Naón, M. J. Salvay, and M. L. Trobo, Phys. Rev. B 70, 195109 (2004).

${ }^{16}$ M. J. Biercuk, N. Mason, and C. M. Marcus, Nano Lett. 4, 1 (2004); M. J. Biercuk, S. Garaj, N. Mason, J. M. Chow, and C. M. Marcus, ibid. 5, 1267 (2005); M. J. Biercuk, N. Mason, J. Martin, A. Yacoby, and C. M. Marcus, Phys. Rev. Lett. 94, 026801 (2005).

${ }^{17}$ E. Goulielmakis, V. S. Yakovlev, A. L. Cavalieri, M. Uiberacker, V. Pervak, A. Apolonski, R. Kienberger, U. Kleineberg, and F. Krausz, Science 317, 769 (2007). 
${ }^{18}$ M. A. Cazalilla, Phys. Rev. Lett. 97, 156403 (2006); C. Kollath, A. M. Läuchli, and E. Altman, ibid. 98, 180601 (2007); M. Cramer, A. Flesch, I. P. McCulloch, U. Schollwöck, and J. Eisert, ibid. 101, 063001 (2008); A. Iucci and M. A. Cazalilla, Phys. Rev. A 80, 063619 (2009).

${ }^{19}$ M. Stone, Bosonization (World Scientific, Singapore, 1994); A. O. Gogolin, A. A. Nersesyan, and A. M. Tsvelik, Bosonization in Strongly Correlated Systems (Cambridge University Press, Cambridge, England, 1998).
${ }^{20}$ D. B. Gutman, Y. Gefen, and A. D. Mirlin, arXiv:0906.4076 (unpublished); Phys. Rev. B 81, 085436 (2010).

${ }^{21}$ E. M. Lifshitz and L. P. Pitaevskii, Statistical Physics Part II (Pergamon Press, Oxford, 1980); G. D. Mahan, Many-Particle Physics, 3rd ed. (Kluwer Academics/Plenum Publishers, New York, 2000); A. Das, Finite Temperature Field Theory (World Scientific, Singapore, 1997); A. Kamenev and A. Levchenko, Adv. Phys. 58, 197 (2009). 